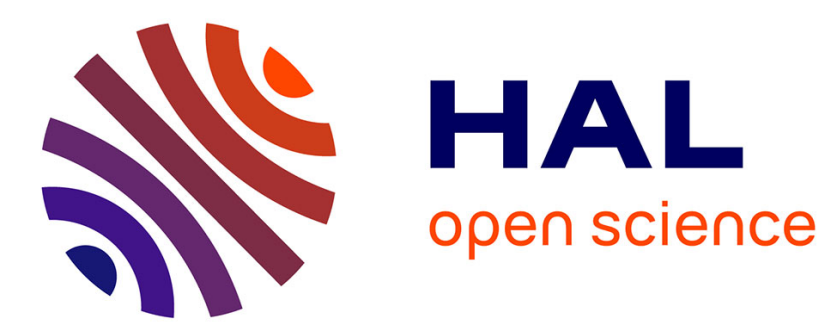

\title{
Bilan en forêt normande
}

Pierre Aubert

\section{- To cite this version:}

Pierre Aubert. Bilan en forêt normande. Revue forestière française, 1963, 6, pp.531-536. 10.4267/2042/24559 . hal-03389951

\section{HAL Id: hal-03389951 \\ https://hal.science/hal-03389951}

Submitted on 21 Oct 2021

HAL is a multi-disciplinary open access archive for the deposit and dissemination of scientific research documents, whether they are published or not. The documents may come from teaching and research institutions in France or abroad, or from public or private research centers.
L'archive ouverte pluridisciplinaire HAL, est destinée au dépôt et à la diffusion de documents scientifiques de niveau recherche, publiés ou non, émanant des établissements d'enseignement et de recherche français ou étrangers, des laboratoires publics ou privés. 


\title{
BILAN EN FORETT NORMANDE
}

\author{
PAR \\ P. AUBERT \\ Ingénieur Principal des Eaux et Forêts \\ à Evreux
}

Dans le numéro de février de cette revue, M. le Conservateur BÉRARD faisait ressortir sous titre de bilan ce qu'ont été pour l'année 1962: production matière, recette totale, revenus brut et net de l'ensemble des forêts du département des Vosges.

A cette lecture, propriétaires et forestiers normands pourraient être subjugués par les résultats ainsi avancés et tentés de croire ces bénéfices réservés aux seuls et heureux tenants des forêts vosgiennes, en tous cas pas aux propriétaires forestiers normands qui feraient figure de parents pauvres dans un pays aux richesses agricoles bien connues.

Pour rassurer ces derniers quant à la productivité possible ou réelle des forêts normandes, nous ne proposerons pas comme exemple les 10600 ha de la célèbre forêt de Lyons car il nous serait objecté à tort ou à raison, qu'il s'agit d'un cas exceptionnel. Nous nous arrêterons plutôt à la modeste forêt de Montfort dont les 2000 ha sont inconfortablement assis sur les bancs de silex qui dominent la basse Risle, entre le gras pays d'Auge et les riches plateaux agricoles du Roumois et du Neubourg. Dans cette région très boisée, le massif de Montfort peut être un bon pilote et la révision d'aménagement en cours est l'occasion de rassembler d'utiles éléments d'information.

La forêt compte actuellement 1000 ha de taillis-sous-futaie et autant de pineraie (sylvestre).

Longtemps ruinée par l'exploitation en taillis aux courtes révolutions de 15 à 22 ans, elle a fait l'objet de divers projets d'aménagement présentés de 1837 à 1865 par un service forestier local soucieux de redresser la situation. L'Administration ne se décida qu'en 1869 pour un aménagement de conversion en futaie régulière aux affectations permanentes, mais déjà la pineraie avait été constituée sur 700 ha des terrains les plus superficiels, caillouteux et dégradés. 
Aujourd'hui, la pineraie âgée pour la plus grande part de 60 à 100 ans est en plein état de production, tandis que la conversion des taillis en futaie a été abandonnée dès 1889 faute d'éléments suffisants dans le taillis, laissant un taillis-sous-futaie plus ou moins

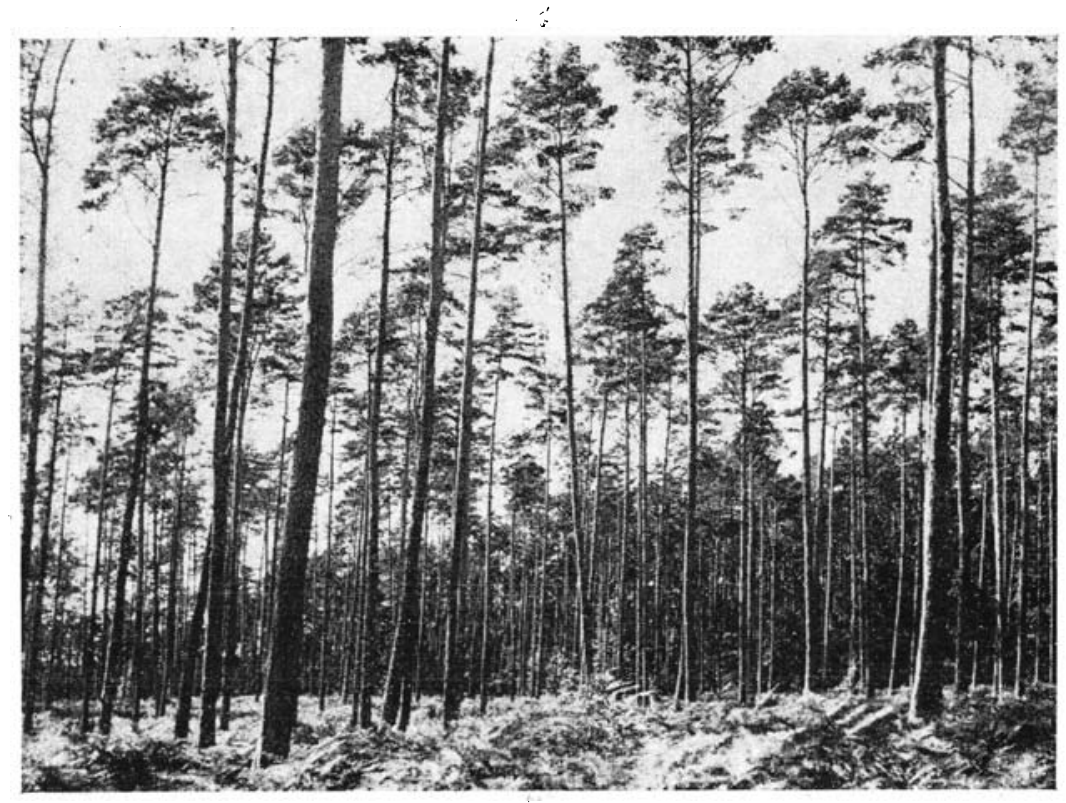

Pineraie en F.D. de Montfort (Eure).

Coupe d'ensemencement.

(Cliché Mazelle.)

enrichi en réserves formant futaie claire dans les meilleurs cantons où le volume de la réserve atteint 150 à $200 \mathrm{~m}^{3}$ par ha cubés au tarif Schaeffer rapide $n^{\circ} 12$. Il est exploité en moyenne chaque année, à côté des éclaircies et régénération de pin, 33 ha de taillis-sousfutaie.

- Quelle production ce massif a-t-il assurée dans le passé, quelle production assure-t-il aujourd'hui?

- La production matière de la forêt durant la période 19271962, soit 36 ans, a été de $4,70 \mathrm{~m}^{3}$ par ha et par an, dont:

$60 \%$ de bois d'œuvre et d'industrie résineux,

$10 \%$ de bois d'œuvre feuillus,

$30 \%$ de feuillus enstérés.

Au cours de la période antérieure, 1912-1926, la production résineuse avait déjà été de $2,17 \mathrm{~m}^{3}$ par ha et par an malgré le manque de maturité de la pineraie. La production d'ensemble pour la 
période 1912-1962 s'établit à 4,50 $\mathrm{m}^{3}$ malgré la charge d'enrichissement du taillis-sous-futaie.

L'intérêt de ces chiffres établis sur une longue période nous parait évident bien qu'ils n'atteignent pas aux rendements dont on fait état aujourd'hui avec les exotiques ou que l'on relève dans les sapinières vosgiennes.

Au cours des trois derniers exercices, cette production a d'ailleurs encore augmenté, atteignant $5,17 \mathrm{~m}^{3}$ surtout en raison de l'intensification des éclaircies résineuses dans des peuplements de pin de plus en plus mûrs.

Pour l'ensemble des forêts soumises du département de l'Eure, la récolte a été en 1962 de $5,50 \mathrm{~m}^{3}$ par ha contre 5,965 $\mathrm{m}^{3}$ dans les Vosges.

Quant au revenu brut et au revenu net de la forêt de Montfort au cours des dix dernières années, ils ressortent du tableau suivant:

\begin{tabular}{|c|c|c|c|c|c|c|c|c|c|c|c|c|}
\hline : & $\begin{array}{l}\text { Exer- } \\
\text { cice }\end{array}$ & $\begin{array}{l}\vdots \\
\vdots \\
:\end{array}$ & $\begin{array}{c}\text { Recette bois } \\
\text { toutes taxes } \\
\text { comprises) } \\
\text { Frs. }\end{array}$ & $\vdots$ & $\begin{array}{l}\text { rt res } \\
\text { rtant } \\
\operatorname{pin}_{c_{j}^{\prime}}\end{array}$ & $\begin{array}{r}\text { au: } \\
\quad:\end{array}$ & $\begin{array}{c}\text { Recette } \\
\text { brute } \\
\text { par ha. } \\
\text { Frs. }\end{array}$ & $\vdots$ & $\begin{array}{c}\text { Recette } \\
\text { nette } \\
\text { Frs. }\end{array}$ & : & $\begin{array}{c}\text { ppel de } \\
\text { produc- } \\
\text { on matiè } \\
\text { re. } \mathbb{r}^{3}\end{array}$ & : \\
\hline : & 1953 & $\vdots$ & 191306 & : & 79 & $:$ & 95 & : & - & : & 3,97 & \\
\hline : & 1954 & $:$ & 201031 & : & 80 & $:$ & 100 & : & 65 & & 4,75 & : \\
\hline : & 1955 & $\vdots$ & 307056 & : & 95 & : & 150 & $\vdots$ & 109 & & 4,54 & $\vdots$ \\
\hline ; & 1956 & $:$ & 180388 & : & 81 & $:$ & 90 & $:$ & 54 & & 3,85 & $:$ \\
\hline 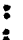 & 1957 & $\vdots$ & 287907 & 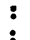 & 75 & $:$ & 144 & : & 98 & & 4,20 & : \\
\hline 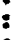 & 1958 & $:$ & 203528 & : & 91 & : & 102 & : & 65 & : & 3,35 & $:$ \\
\hline & 1959 & $:$ & 205558 & : & 88 & : & 103 & & 58 & : & 3,71 & $:$ \\
\hline 8 & 1960 & $\vdots$ & 275633 & : & 85 & $\vdots$ & 138 & : & 98 & & 5,11 & 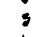 \\
\hline$:$ & 1961 & $:$ & $400 \quad 057$ & : & 86 & $:$ & 200 & & 144 & & 5,28 & $:$ \\
\hline : & 1962 & $:$ & 528204 & $\dot{\Sigma}$ & 85 & $\vdots$ & 264 & : & - & $\vdots$ & 5,11 & $\vdots$ \\
\hline
\end{tabular}

Le revenu net est établi chaque année sous forme d'un état de rentabilité pour chacune des forêts de la $2^{\mathrm{e}}$ Conservation; il tient compte de toutes les recettes, bois et autres, et de toutes les dépenses d'entretien et de personnel.

Ce revenu net ressort en moyenne à $70 \%$ du revenu brut. Dans les $30 \%$ de frais, le personnel figure pour $18 \%$ et les travaux pour $12 \%$.

On remarquera la place largement prépondérante, $85 \%$, tenue par le pin parmi les éléments du revenu, bien qu'il ne représente que $60 \%$ de la production matière.

L'augmentation du revenu est dù à trois facteurs:

- augmentation de la production matière,

- prépondérance du pin,

- augmentation du prix du pin. 
De 1957 à 1962, le prix du $\mathrm{m}^{3}$ de pin, toutes taxes comprises, est passé de 68 à $80 \mathrm{~F}$ pour les arbres de $1 \mathrm{~m}^{3}$, de 48 à $60 \mathrm{~F}$ pour les arbres de $0,6 \mathrm{~m}^{3}$ en moyenne.

Ce prix, sa variation en fonction de la grosseur de l'arbre et au cours des cinq dernières années, l'évolution de la production de la pineraie, et sa répartition entre produits d'éclaircie et produits principaux, sont donnés selon graphiques joints.

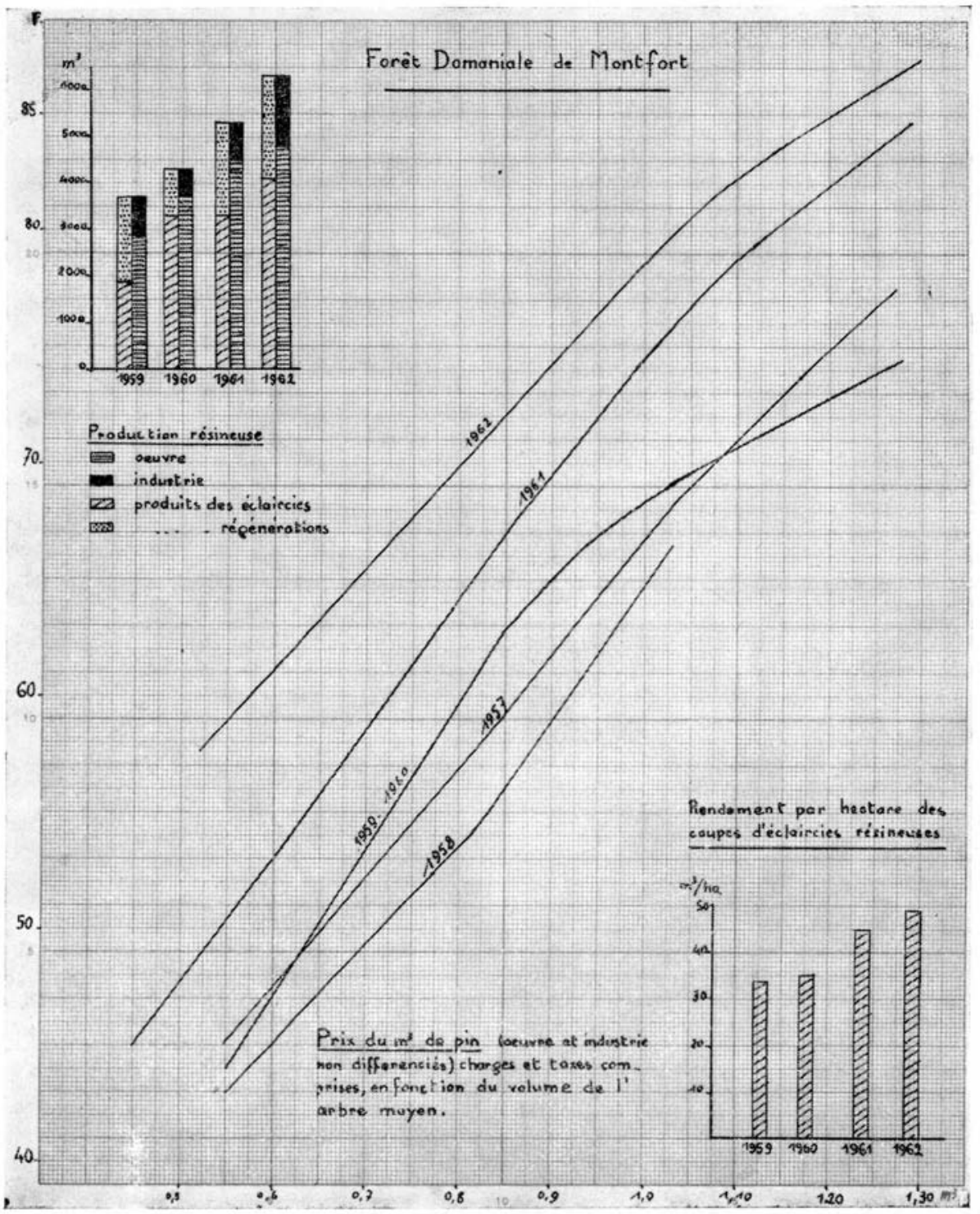


Le revenu net à l'ha qui atteint $150 \mathrm{~F}$ par ha alors que la moitié de la forêt est encore en taillis-sous-futaie, est appelé à augmenter au cours de la prochaine période car il va falloir accélérer la réalisation de la pineraie de première génération et procéder également à des réalisations dans la réserve de taillis-sous-futaie pour introduire à côté d'un élément feuillu indispensable (le hêtre), des résineux exotiques à croissance rapide.

Alors, à force de soins, avec le concours d'investissements limités (100 hectares d'enrésinement prévus à l'actuel plan quadriennal) la production de cette forêt deviendra comparable à celle de la forêt vosgienne qui bénéficie, grâce à son altitude, d'un climat aussi favorable que le climat océanique bien qu'avec une saison plus courte, mais a comme autre atout l'avantage, du moins dans sa partie granitique, de sols beaucoup mieux partagés chimiquement.

Les chiffres avancés ci-dessus l'ont été au titre de récolte plutôt que de production. Il importe en effet de distinguer les deux termes et de préciser. Pour l'ensemble des forêts soumises du département, la récolte ne paraît guère s'écarter de la production en l'état actuel des peuplements, ne serait-ce que du fait de la part primordiale qu'y occupe la forêt de Lyons, forêt aménagée par affectations permanentes dans lesquelles l'ordre atteint à la plus grande rigueur. Dans le cas de Montfort qui nous retient particulièrement, le déséquilibre entre les classes d'âge et la masse des pineraies arrivant à l'âge de réalisation vont provoquer une augmentation de récolte; celle-ci pourrait ne plus être en rapport avec une production soutenue. A une période de capitalisation dans des pineraies vieillissantes va succéder une période de réalisation et de rajeunissement. Mais dans le cas du pin sylvestre, à l'opposé de ce que l'on constate avec le sapin de Normandie, la croissance est très rapide dans la première moitié de l'âge et lente ensuite (50-100 ans), d'où l'effet stimulant sur la production d'une récolte accélérée des vieux bois. D'ailleurs, les rendements relevés ci-dessus concernent la période écoulée, non celle des réalisations à venir, ils constituent donc de sérieux éléments de comparaison pour l'appréciation de la productivité des forêts normandes.

Face à la séduisante renommée des forêts vosgiennes, les propriétaires et forestiers normands n'ont donc pas à s'attarder à la contemplation mélancolique des landes à fougère parsemées de bouleau, si jolies qu'elles soient sous le soleil de mars ou de no-1 vembre, mais plutôt à s'attacher à leurs hêtraies, à leurs pineraies, aux plantations d'exotiques faites par leurs aieux, telle cette pineraie de Laricio de Corse située sur une butte sableuse près de Vernon dans laquelle la Station de Recherches Forestières a inventorié $897 \mathrm{~m}^{3}$ de bois fort par ha avec une production estimée à $10 \mathrm{~m}^{3}$ par ha et par an.

Ces exemples nous rappelleraient, s'il était nécessaire, que la forêt est ce que l'homme la fait. 
Les 18000 ha de forêt soumise du département ont produit, nous l'écrivons ci-dessus, 5,50 $\mathrm{m}^{3}$ par ha en 1962; les 110000 ha de forêts privées doivent produire autant. De plus en plus nombreux sont les propriétaires qui l'ont compris, ils s'attachent activement à le réaliser. 